\title{
REDES SOCIAIS, VALORES E COMPETÊNCIAS: SIMULAÇÃO DE CONEXÕES
}

RESUMO

Este artigo tem por objetivo desenvolver um al goritmo para representar a dinâmica das interações que conduzem à formação de conexões entre indivíduos dentro de uma organização. Por meio de um procedimento de simulação computacional, utiliza-se um critério matemático para a definição da opinião de um indivíduo sobre outro. 0 intuito éidentificar como diferentes características, em especial o consenso sobre as competências de um indivíduo e o peso atribuído aos valores pessoais, influem na estrutura das redes sociais. Os resultados do modelo mostram que, quando o consenso é alto, a rede é mais densa, conduzindo a relacionamentos mais complexos dentro da organização, podendo implicar sinergias e até mesmo inércia. De modo contrário, quando o consenso é baixo, conflitos em pequenos grupos podem constituir barreiras para a comunicação entre os indivíduos.

\section{Herbert Kimura}

Universidade Presbiteriana Mackenzie

\section{Maria Luisa Mendes Teixeira}

Universidade Presbiteriana Mackenzie

\section{Arilda Schmidt Godoy}

Universidade Presbiteriana Mackenzie

\begin{abstract}
ABSIRACTThis article intends to develop an algorithm that represents the dynamics of the interactions that lead to the establishment of ties between individuals within a group. Through a procedure involving a computer simulation, a mathematical criterion for the scoring of opinions related to an individual is defined, in order to identify how different characteristics, in special the consensus on the skills of an individual and the weight attributed to personal values, influence the structure of social networks. Results show that, when consensus on skills is high, the network has more density, leading to more complex relationships within the organization, and eventually implying synergies and even inertia. Conversely, when the consensus is low, conflicts in small groups might be communication barriers among individuals.
\end{abstract}

PALAVRAS-CHAVE Redes sociais, difusão de opinião, competências e valores, modelo matemático de redes, simulação computacional. KEYWODS Social networks, diffusion of opinion, skills and values, mathematical model of networks, computer simulation. 


\section{INTRODUÇÃO}

A análise de redes sociais tem sido objeto de estudo de pesquisas acadêmicas bem como de aplicação por consultorias em gestão (Borgatti e Molina, 2003). A partir das experiências seminais de Milgram (1967) sobre cadeias de relacionamento mútuo, o estudo quantitativo de redes sociais despertou 0 interesse de acadêmicos e a curiosidade popular. Borgatti e F oster (2003) destacam o crescimento exponencial da literatura sobre redes, enquanto Granovetter (1985) cita que pesquisas em teoria de Administração têm utilizado cada vez mais premissas da integração do indivíduo em redes sociais.

Com o aumento da capacidade de processamento numérico, a abordagem computacional para o estudo de redes sociais tem possibilitado que sistemas mais sofisticados sejam simulados e avaliados em maiores detal hes. 0 desenvolvimento da informática propicia um campo de estudo alternativo da dinâmica das redes sociais, uma vez que as características dos grupos podem ser simuladas em um ambiente artificial sem a necessi dade de observação de atitudes reais, facilitando o processo de anál ise de sensibilidade das redes em função de al terações em parâmetros do modelo. Nesse sentido, o modelo teórico pode apresentar vantagens, pois, dada a complexidade da formação de conexões em uma rede real, variáveis observáveis podem conter ruídos sujeitos a inúmeros fatores que prejudicam as análises provenientes de estudos empíricos.

Exemplificando, análises empíricas podem envolver respostas a questionários que eventualmente podem não representar adequadamente os constructos de interesse. Mais ainda: as condições de aplicação do questionário podem ser pouco controladas, implicando 0 aumento da variabilidade da variável observada e prejudicando a significância dos resultados. Adicional mente, podem-se realizar observações empíricas em escala inapropriada para a utilização de várias técnicas estatísticas.

Nesse cenário de limitações da análise empírica, estudos teóricos baseados em mecanismos de simulação matemática auxiliam a formulação de hipóteses. Por exemplo, Abrahamson e Rosenkopt (1997) utilizam a simulação computacional para estudar efeitos das redes sociais, enquanto Harrison e Carroll (2002) analisam o comportamento de redes de influência ao longo do tempo por meio de um algoritmo baseado em um modelo de transmissão cultural em organizações.
Considerando a perspectiva de modelagem, este artigo tem por objetivo desenvolver um algoritmo para representar a dinâmica das interações que conduzem à formação de conexões entre indivíduos dentro de uma organização. Por meio de um procedimento de simulação computacional, utiliza-se um critério matemático para definir a opinião de um indivíduo sobre outro, com 0 intuito de identificar como diferentes características - em especial, o consenso sobre as competências de um indivíduo e o peso atribuído aos valores pessoais - influenciam a formação de redes.

Definiram-se no modelo os determinantes da conexão de indivíduos em um grupo, considerando variáveis aleatórias como proxy de aspectos objetivos associados às competências de um indivíduo, e de aspectos subjetivos associados aos valores pessoais. Adicional mente, considerou-se um parâmetro de distância geográfica ou dificuldade de comunicação entre indivíduos que também influencia na possibilidade de interação.

Apesar da suposição de um ambiente artificial com diversas premissas simplificadoras, o model o permite a avaliação do mecanismo de formação de redes no ambiente corporativo. A pesquisa aborda uma perspectiva de modelagem para a análise de redes, fundamentada no uso de procedimentos computacionais, que não é comumente utilizada em trabalhos da área de Ciências Sociais aplicadas no Brasil. A modelagem, a priori acompanhada de processos de simulação, traz como vantagem a possibilidade de geração de hipóteses e reflexões a elas associadas, possibilitando uma relação dialética com a realidade empírica, visando a construção de teoria. Outra vantagem da modelagem teórica diz respeito à possibilidade imediata de estimular esforços simultâneos de pesquisa e discussão sobre o modelo, promovendo o seu amadurecimento decorrente de diversos aportes empíricos.

\section{REFERENCIAL TEÓRICO}

\section{Abrangência das redes sociais}

De acordo com Brass (1984), a perspectiva das redes sociais está ligada à idéia de que as interações sociais aprimoram ou restringem 0 acesso a recursos. Conforme estabelece Collier (1998), a interação social incorpora um capital social e pode gerar três externalidades: o conhecimento sobre o comportamento dos outros agentes; o conhecimento sobre o mercado, como preços e tecnologias; e o benefício da ação coletiva. 
Putnam (1993) conceitua capital social como um conjunto de associações horizontais entre pessoas que consiste em redes sociais imbuídas de normas com efeito na produtividade de uma comunidade. Para Leana e Buren (1989), o capital social organizacional representa um recurso que reflete o caráter das relações sociais existentes na organização, decorrente da orientação coletiva para al cançar os resultados e da confiança compartilhada por seus membros. 0 capital social organizacional, por ser desenvolvido em redes sociais, possui natureza complexa e de difícil reprodução, constituindo-se em fonte de vantagem competitiva.

As redes sociais, lócus do capital social, para serem formadas, dependem de conexões. Alguns parâmetros quantitativos têm especial importância para identificar a estrutura de uma rede social. A densidade descreve o nível geral de interações apresentadas pelos membros de uma rede, sendo associada ao número médio de conexões por membro do grupo. A centralização reflete a concentração de conexões em um número reduzido de indivíduos, em contraposição a uma distribuição mais igualitária, estando associada à variância do número de conexões por indivíduo (Sparrowe, Liden e Kraimer, 2001).

De acordo com Portes e Sensenbrenner (1993), a densidade da rede tende a fomentar a identificação do grupo, facilitando a troca de experiências e as ações coletivas, devido a uma maior relação de confiança mútua (Coleman, 1988). Quando os agentes desenvolvem conexões com grupos desconectados, diminuindo a centralização, ganham acesso a informações, diminuindo a redundância dos dados (Reagans e Zuckerman, 2001) e fazendo com que a difusão de conhecimento possa conduzir ao aproveitamento de um maior número de oportunidades. Dessa forma, uma diversidade de competências, informação e experiência podem eliminar falhas estruturais das organizações, aprimorando sua capacidade de ação criativa (Ancona e Caldwell, 1992).

A partir do levantamento de trabalhos recentes, pode-se verificar a abrangência dos estudos acerca das redes. De uma perspectiva social, Armengol e Jackson (2004) estudam os efeitos das redes sobre desigualdade e empregabilidade. Murphy e Shleifer (2004) desenvolvem um modelo associado à criação de redes sociais e seu uso político. Cummings (2004) sugere que o compartilhamento de conhecimento externo é fortemente relacionado com desempenho quando os grupos de trabal ho têm uma maior diversidade estrutural.
Numa abordagem voltada ao contexto empresarial, Podolny e Baron (1997) sugerem que redes informais possibilitam a troca de recursos como experiências e a difusão de informações estratégicas associadas ao trabal ho, permitindo a definição de normas e identidade de uma empresa. Com relação à dinâmica de negócios, Rauch (2001) apresenta evidências de que as redes sociais promovem o comércio internacional por reduzirem problemas de execução de contratos e de difusão de informações. A formação de conglomerados e de alianças corporativas também pode ser estudada por meio de conceitos de redes, como em Sorenson (2003). $\mathrm{N}$ o contexto de proximidade de relacionamento empresarial, Jones, Hesterly e Borgatti (1997) estabelecem a existência de conexões sociais devido à vantagem competitiva que se obtém por meio da proteção de transações econômicas.

Considerando o avanço tecnológico, Wejnert (2002) desenvolve uma estrutura conceitual baseada em redes sociais para explicar a difusão de tecnologias. Além disso, novas configurações de trabal ho, como a colaboração on-line, podem ter um componente de rede de relacionamento que impulsiona a produtividade. Herbsl eb e Mockus (2003) estudam o desenvolvimento global de software, testando hipóteses que envolvem características de redes sociais distribuídas geograficamente, e Leenders, Engelen e Kratzer (2003) abordam a comunicação, a virtualidade e a criatividade de equipes a partir de uma perspectiva de redes sociais. Borgatti (2003) sumariza as diversas áreas de aplicação da teoria de redes sociais: Iiderança, desempenho no trabalho, empreendedorismo, relações entre stakeholders, uso do conhecimento, inovação, maximização de lucro, colaboração entre empresas etc.

A intensidade das redes e a confiança mútua dependem do consenso dos indivíduos que nelas interagem quanto à opinião que possuem sobre os demais, formada a partir da percepção sobre o outro, a qual reforçará, ou não, o comportamento de cada um. Entre as perspectivas para a compreensão do comportamento dos indivíduos em grupo, tendo como foco a interação, M cLeod e Chaffee (1973) apontam o consenso. Wirth (1948) entende que se trata de um estado de opinião relativamente homogênea entre um conjunto de indivíduos. 0 consenso é extremamente importante numa sociedade muito fragmentada, em que os métodos de gestão top-down não mais se mostram efetivos e é necessário buscar soluções criativas (Susskind, 1999), além de promover a cooperação, o comprometimento e o compartilhamento de visões (Berman e Werther, 1996). 
Tomando por base M cLeod e Chaffee (1973), assume-se neste trabal ho uma abordagem interpessoal para a compreensão da formação das redes sociais, onde 0 comportamento dos indivíduos é uma função da sua percepção das orientações dos outros com quem convivem no grupo e da sua orientação para com eles, constituindo-se o consenso numa perspectiva de estudo, nessa abordagem.

De acordo com Wooldrige e Floyd (1989), o consenso apresenta três dimensões: grau, escopo e conteúdo. 0 grau representa o quanto as pessoas concordam entre si, o escopo diz respeito a quem participa do consenso, e o conteúdo se refere ao objeto do consenso. 0 modelo desenvolvido neste artigo não contempla, em sua análise, o grau do consenso, restringindo o escopo a situações em que as pessoas não têm conhecimento mútuo prévio antes de iniciarem um processo de interação, e o conteúdo a dois constructos, aqui considerados como "parâmetros do modelo": valores e competências.

\section{Parâmetros usados no modelo para a formação das redes}

No modelo proposto neste artigo, as conexões da rede dependem da formação de opinião sobre os indivíduos da sociedade. Basicamente, duas características são consideradas no processo de atribuição de opiniões: competências, de natureza observável; e valores pessoais, de natureza não observável. A despeito da abrangência dos temas, considerando o foco do trabalho, será realizada uma breve discussão sobre competências e valores.

A noção de competências na área de organizações e gestão tem recebido atenção desde a década de 1970, quando o termo passou a ser entendido como o conjunto de qualificações que a pessoa possui para executar seu trabalho com um nível superior de desempenho. Entretanto, esse conceito foi posteriormente criticado, complementado e modificado, sendo possível identificar atualmente duas grandes tendências nessa área: a dos autores anglo-saxões, que adotam uma abordagem mais pragmática, e dos franceses, que incorporam elementos da sociol ogia e da economia do trabal ho em sua análise (Cariola e Quiroz, 1997).

Neste artigo, a vertente da escola francesa é adotada como referência. Para os franceses, como Le Boterf (2003), é preciso distinguir entre dois modelos de competências que interferem atualmente nas práticas de gestão: um apoiado nas concepções tayloristas e fordistas, outro, tomado como referência neste trabaIho, em que o sujeito é visto como um ator que sabe ir além do prescrito, que sabe agir e tomar iniciativas. Considera-se neste artigo que existem maneiras de ser competente e que diferentes condutas podem ser pertinentes, estando a competência associada à faculdade de desencadear e de conjugar recursos e ações, conforme apontam, na literatura nacional, Dutra (2004), Fleury e Fleury (2001) e Ruas (2005).

Zarifian (2003, p. 137) estabelece seu conceito de competência a partir das críticas feitas à organização taylorista, definindo-o a partir de três el ementos complementares: (a) competência é a tomada de iniciativa e responsabilidade do indivíduo em situações profissionais com as quais ele se defronta; (b) competência é uma inteligência prática das situações, que se apóia em conhecimentos adquiridos e os transforma à medida que a diversidade das situações aumenta; (c) competência é a faculdade de mobilizar redes de atores em volta das mesmas situações, de compartilhar desafios, de assumir áreas de responsabilidade.

De acordo com a noção de competência proposta por Zarifian (2001, p. 74), quando uma situação de trabal ho se torna mais complexa as competências de um único indivíduo se tornam insuficientes, havendo a necessidade da articulação de uma rede de atores. Exige-se de cada in divíduo que ele perceba que necessita de competências que não possui, mas que poderão ser adquiridas a partir da solidariedade de uma ação conjunta, tornando coletivas as situações de trabalho. É possível encontrar movimentos nesse sentido quando o trabal ho é organizado em equipe, em rede ou por projetos.

Além disso, à responsabilidade individual se alia a questão da co-responsabilidade. É nessa situação de trabal ho, na qual temos um grupo de pessoas engajadas em uma mesma prática de trabal ho, que se apresenta a questão do compartilhar certos valores, "pois só se pode associar responsabilidade social e responsabilidade coletiva se cada sujeito aceita desenvolver certa moral de comportamento" (Zarifian, 2001, p. 76). Assim, 0 indivíduo estabel ece uma rel ação entre o conjunto de atos que ele realiza em seu trabal ho e o sistema de valores que orienta esses atos, o que também é detectado por M CLagan (1997). Para Zarifian (2001), cabe aos indivíduos que pertencem a uma determinada área de trabalho, em uma cultura específica, estabelecer um consenso - explícito ou implícito - em torno de um determinado conjunto de valores aos quais aderir. Enquanto valores impostos podem ser rapidamente rejeitados, valores em torno dos quais haja um consenso podem ser exteriorizados e formalizados. 
Evidentemente, a influência desses valores na atividade profissional deve contar ainda com o seu alinhamento à eficiência produtiva da empresa.

Segundo Schwartz (1992), existe na literatura sobre valores um acordo acerca de sua definição, qual seja: valores são crenças que pertencem a fins desejáveis ou formas de comportamento; que transcendem situações específicas; que guiam a seleção ou avaliação de comportamentos, pessoas e eventos; e que se ordenam por importância relativa a outros valores, formando um sistema de prioridades de valores.

Os valores servem como princípios que orientam a vida de uma pessoa ou de uma entidade social (Schwartz, 1992). Dessa forma, os valores atendem aos interesses de al guma entidade social, podem motivar a ação, funcionam como critérios para julgar e justificar a ação e são adquiridos tanto por meio da socialização nos valores do grupo dominante como por meio da experiência pessoal. Para o autor, os valores são a expressão das necessidades inerentes à existência humana dos grupos sociais e dos indivíduos em função do processo de adaptação à realidade de um determinado contexto social. Os valores representam, em forma de metas conscientes, a resposta dos indivíduos e da sociedade a três requisitos universais: necessidades dos indivíduos enquanto organismos biológicos; mecanismos de interação social coordenada; e requisitos para o correto funcionamento e sobrevivência dos grupos.

Schwartz (1992) estrutura os valores pessoais em duas dimensões bipolares opostas: autopromoção versus autotranscendência, e abertura à mudança versus conservação. A primeira refere-se à contraposição entre a orientação para si mesmo ou para o outro, e a segunda à oposição entre a valorização da independência da ação ou dos costumes, da segurança e normas sociais. A cada um dos pólos dessas dimensões se relacionam conjuntos de valores que mantêm entre si uma relação de compatibilidade. À autopromoção estão associados os valores poder e realização; à abertura para trocas, o hedonismo, a estimulação e a autodeterminação; à autotranscendência estão associados 0 universalismo e a benevolência; e finalmente a conservação, englobando a tradição, a conformidade e a segurança.

Tanto as dimensões bipolares quanto os valores que delas fazem parte assumem hierarquias diferentes de acordo com a importância que Ihes é atribuída pelos indivíduos. A hierarquia é capaz de explicar, ainda que em parte, a afinidade entre os indivíduos e a tolerância na convivência entre si, para o que é necessário que priorizem o mesmo pólo. Assim, indivíduos que priorizam a conservação e não priorizam a abertura à mudança podem apresentar maior afinidade entre si, e maior facilidade de consenso, assim como aqueles que, ao contrário, valorizam a abertura à mudança, 0 mesmo ocorrendo em relação ao pólo autopromoção e autotranscendência. Quanto mais os indivíduos que fazem parte das redes sociais priorizarem os mesmos pólos das dimensões de valores, tanto mais fácil será o consenso entre eles, quanto aos diversos temas da vida organizacional. 0 contrário - a priorização diferente dos mesmos pólos - pode não só dificultar o consenso, mas também antagonismo, como, por exemplo, quando um indivíduo valoriza a conservação e outro, a abertura à mudança.

Diferentemente das competências, que são traduzidas em comportamentos, os valores de um indivíduo, por serem não observáveis e cuja priorização é passível de identificar quando há necessidade de decidir entre al ternativas (Schwartz, 1992), exigem, para serem percebidos por outros indivíduos, tempo de interação, Assim, as conexões entre os indivíduos numa rede social podem ser facilitadas pela percepção das competências. Posteriormente, poderão ou não ser estimuladas pela percepção das prioridades dos valores individuais, podendo eventualmente reforçar ou não a confiança mútua e o consenso.

\section{DINÂMICA DO MODELO}

0 modelo matemático desenvolvido busca representar a interação entre os indivíduos dentro de um grupo e seus possíveis impactos. Incorpora alterações na modelagem proposta por Zinchenko et al. (2003), para melhor adequação à realidade, principalmente no que se refere à dinâmica de formação de conexões entre indivíduos e à reavaliação de opinião em função dos contatos de cada indivíduo.

Basicamente, cada in divíduo apresenta características que constituem parâmetros mediante os quais outros indivíduos formam uma opinião. Os indivíduos formam opinião sobre outros indivíduos baseando-se em suas percepções sobre as características dos integrantes do grupo com os quais interagem. Considerase a opinião como função de duas dimensões. A primeira dimensão, considerada objetiva por ser observável, está ligada às competências do indivíduo. A segunda dimensão, subjetiva, por não ser observável é associada aos valores dos indivíduos. 
Apesar de os termos "objetivo" e "subjetivo" serem controversos nas Ciências Sociais, neste modelo as competências e os valores são definidos como características de cada indivíduo e serão refletidos matematicamente em variáveis que exprimam a opinião que cada indivíduo tem sobre outro. A seguir, o modelo é descrito em maiores detalhes, apresentando-se suas premissas básicas.

\section{Ambiente e agentes}

Considere-se um grupo composto por um número fixo $\mathrm{N}$ de indivíduos que, inicialmente, não possuem contato entre si. Sem perda da generalidade, o foco do modelo envolve grupos de pessoas que interagem no ambiente de negócios, dado o potencial de aplicação do conceito de redes para o meio empresarial.

É importante destacar que o modelo permite a identificação da dinâmica da construção de conexões entre indivíduos que, no instante inicial, não possuem nenhuma ligação entre si. Assim, grupos já formados, com conexões pré-existentes, poderiam ser casos especiais do modelo apresentado neste trabal ho.

No modelo, atribuem-se a cada indivíduo dados numéricos correspondentes às competências e aos valores no instante em que o grupo é formado. De certa maneira, o modelo incorpora a distinção usualmente utilizada pelos estudiosos de redes sociais, como Fombrun (1982), que estabelece uma separação entre laços instrumentais e laços expressivos. Os laços instrumentais estariam associados às atividades realizadas no trabalho, podendo-se considerar um lócus de competências, enquanto os laços expressivos estariam associados a um senso de identidade individual, do qual fazem parte os valores.

Para fins de simulação de realização de variáveis aleatórias associadas a essas características em uma população, atribui-se a cada indivíduo um nível intrínseco de competências e de valores. Estabelece-se, para fins de simulação, que as competências têm uma distribuição normal, enquanto os valores possuem uma distribuição uniforme.

\section{Escore inicial}

No modelo, cada indivíduo possui uma opinião latente, ou escore inicial, baseada nas características dos indivíduos, quanto a competências e valores. 0 escore inicial constitui dado fundamental para a definição das conexões da primeira iteração do model o e base para a opinião a ser considerada quando uma primeira conexão entre dois indivíduos é estabelecida em iterações subseqüentes.
As opiniões sobre um mesmo indivíduo podem divergir principalmente em relação aos aspectos subjetivos. 0 modelo incorpora um redutor, denominado parâmetro de tolerância, que diminui o impacto negativo da diferença entre a hierarquia de valores dos indivíduos. Esse redutor representa a tolerância das pessoas para interagirem com outras que defendam uma hierarquia de valores diferente da sua.

Os aspectos objetivos são menos sujeitos a divergências, pois os resultados das competências são mais evidentes. Assim, nenhum parâmetro de ajuste das competências é considerado no modelo. Existe uma diferença entre as características que os indivíduos possuem e aquelas percebidas pelos outros. No model o, essa diferença foi denominada "ruído" ou "erro". Esse é um ponto importante, pois o erro diferencia percepções sobre um mesmo indivíduo, independentemente dos pesos relativos atribuídos às competências (dimensão objetiva) e val ores (dimensão subjetiva) denotados pelo parâmetro de consenso. Por exemplo, uma mesma pessoa pode ter valores diferentes de competências associados por diferentes indivíduos. Deve-se observar que o termo "erro" é análogo ao seu conceito estatístico, no qual uma estimativa apresenta uma diferença em relação ao verdadeiro valor de uma variável.

Estabelece-se que os ruídos de percepção, ou erros associados às percepções das competências e dos vaIores, têm uma distribuição normal . 0 erro de percepção de i sobre as competências de é é dado por $\operatorname{Erro}_{H}(i, j)$ enquanto o erro de percepção de i sobre os valores de j é dado por $\operatorname{Erro}_{v}(\mathrm{i}, \mathrm{j})$. Matematicamente, adaptando o modelo de Zinchenko et al. (2003) para o propósito deste trabalho, a opinião latente ou Escore(i,j) que um indivíduo i atribui a um indivíduo j é dada por:

$$
\begin{aligned}
\operatorname{Escore}(i, j)= & \text { Consenso }\left[\text { Competência }(j)+\operatorname{Erro}_{H}(i, j)\right]+ \\
& (1-\text { Consenso })\left[\left(\operatorname{Tolerância~}+\operatorname{Erro}_{V}(i, j)\right)-\right. \\
& \mid \text { Valores }(i)-\operatorname{Valores}(j) \mid]
\end{aligned}
$$

Portanto, quanto maior a competência de je menor a diferença de valores entre i e j, maior o escore atribuído pelo indivíduo i ao agente j. 0 aspecto objetivo implica que, quanto maior a competência do indivíduo j, melhor a percepção que se tem de j. 0 aspecto subjetivo implica que, quanto mais valores em comum os indivíduos i e j têm, melhor a percepção que um tem do outro. Os erros associados às competências e aos valores decorrem da impossibilidade de um indivíduo conseguir fazer uma leitura totalmente acurada das características intrínsecas de outro. 
Dentro do conjunto de opiniões latentes que um indivíduo atribui aos outros indivíduos do grupo, pode-se obter o escore médio e o desvio padrão dos escores de i, por meio das respectivas fórmulas:

$\mu_{\text {Escore }}(i)=\frac{\sum_{k=1, k \neq i}^{N} \operatorname{Escore}(i, k)}{N-1}$ e $\sigma_{\text {Escore }}(i)=\sqrt{\frac{\sum_{k=1, k \neq i}^{N}\left(\text { Escore }(i, k)-\mu_{\text {Escore }}(i)\right)^{2}}{N-1}}$

\section{Posição inicial dos indivíduos}

0 modelo também estabelece uma local ização individual dos integrantes do grupo. Em uma corporação, essa distância pode estar associada à localização geográfica. No al goritmo, define-se que a localização inicial dos indivíduos é distribuída uniformemente em duas dimensões, evitando assim uma situação inicial em que conglomerados já existam, fato que poderia orientar as conexões da rede em função da concentração preliminar de indivíduos. Assim, no modelo, cada indivíduo tem inicialmente uma posição distribuída em linhas e colunas. A distância entre i e j, em termos físicos ou de comunicação, é dada por:

Distância(i,j) =|Linha(i) - Linha(j) $|+|$ Coluna(i) - Coluna(j)|

Observe-se que a distância não é euclidiana, representando apenas um distanciamento entre os indivíduos, medida pelo menor número de passos que um indivíduo i deve seguir em um espaço ortogonal de duas dimensões para atingir a posição do indivíduo j. Dessa forma, por meio do modelo, indivíduos muito distantes não formarão diretamente conexões entre si, pois a confiabilidade de suas opiniões preliminares seria muito prejudicada pela distância.

\section{Formação de conexões entre os indivíduos}

A pesar de o modelo estabelecer uma opinião latente de um integrante do grupo sobre o outro, as conexões da rede podem não envolver todos os indivíduos. Duas condições são necessárias para que haja conexão do indivíduo i para o indivíduo j. A primeira condição estabelece que os indivíduos não podem ter uma distância superior a um limite ou referência de distância (RD). Se a distância entre dois indivíduos é maior que $R D$, então a conexão inexiste. Dessa maneira, se RD é alta, menor a probabilidade de a distância entre os indivíduos ser maior que RD e maior a probabilidade de haver conexão. Assim, RD el evada pode representar empresas concentradas geograficamente em uma região, ou empresas em que a comunicação entre os in- divíduos é ampla. Nessas situações, torna-se mais provável que dois indivíduos interajam e possam transformar opiniões latentes em opiniões efetivas. 0 caso em que RD é pequena representa empresas com uma ampla distribuição geográfica, possuindo várias filiais no mundo, ou empresas com mecanismos de comunicação ou estruturas organizacionais que dificultam a interação entre os funcionários.

A segunda condição para a formação de conexões na rede está associada ao escore atribuído a um indivíduo. Se o escore, ou a opinião de i sobre j, for al to, maior a probabilidade de se formar uma conexão de i para j. No modelo, cada indivíduo atribui escores latentes para todos os outros, porém não se forma necessariamente uma conexão. Somente quando um indivíduo i forma uma opinião razoavelmente positiva sobre 0 indivíduo j, há a propensão ao estabelecimento de uma conexão de i para j.

U ma opinião é considerada razoavel mente positiva quando i atribui a j um escore superior a uma referência de escore (RE). Essa referência de escore representa o número mínimo de desvios padrão $\sigma_{\text {Escore }}(i)$ acima da média da opinião que i deve atribuir a j para que i tenha motivação suficiente para querer se conectar a j.

Assim, se 0 individuo j tiver um escore, na percepção de i, superior ao limite mínimo RE, então há uma possibilidade de con exão entre i e j. O s indivíduos são propensos a criar conexões com pessoas com escores altos, ou seja, com pessoas que estimam ou que respeitam em função de uma opinião formada a partir da percepção das competências e dos valores dos outros.

\section{Interação entre indivíduos}

As opiniões de um indivíduo j sobre k podem ser compartilhadas por todos os indivíduos i que se conectem a j. Mesmo não havendo uma conexão direta, a rede de relacionamento de i impacta também o escore atribuído a um dado indivíduo $\mathrm{k}$. Em termos dinâmicos, um indivíduo i pode perguntar a um agente conectado j a opinião de j sobre um outro indivíduo k. Dessa maneira, o agente $i$, sem conhecer diretamente $k$, pode formar uma opinião mais elaborada sobre $k$ baseando-se não somente em sua opinião latente como também na opinião de outros indivíduos de sua rede de relacionamento.

Nesses casos, o indivíduo i pondera a opinião própria sobre k e a opinião de terceiros sobre k de maneira diferenciada. No modelo, quanto maior o fator de ponderação, maior o peso dado à opinião própria. A opinião de terceiros sobre um individuo k é um agregado das opiniões sobre $k$ de todos os indivíduos j aos 
quais i se conecta. No modelo, cada opinião de j sobre k é ponderada por um parâmetro relativo às diferenças de valores entre i e j. Dessa maneira, quanto mais próximos Valores(i) e Valores(j), maior o peso atribuído por i à opinião de j sobre k. Com isso, o modelo permite que cada indivíduo pondere a opinião de terceiros em função de um parâmetro de afinidade.

Além da troca de opinião entre os indivíduos na sociedade, o modelo incorpora um mecanismo de realimentação positiva. Se o escore agregado de terceiros sobre $k$ for maior do que o escore atribuído por i a k, então o escore final de i sobre k terá uma correção aleatória positiva. Assim, após a interação, o Escore( $i, k)$ sofre um aumento al eatório. Se o escore agregado de terceiros for menor do que o escore atribuído por i a k, então existe uma correção negativa, e, dessa maneira, há uma penalização do escore final do indivíduo k percebido por i. Esse fator de correção tem uma distribuição uniforme.

\section{Correção da posição dos indivíduos}

À medida que ocorrem interações e se criam conexões, os indivíduos eventualmente se aproximam ou se distanciam no espaço de duas dimensões, corrigindo suas posições de linhas ou colunas. Quando se forma uma conexão de i para j, o indivíduo i aproxima-se do indivíduo j com uma probabilidade arbitrária Prob $_{\text {Aprox }}$ corrigida por um grau de afinidade dos valores, ajustando sua posição na linha ou na coluna com probabilidade de 0,5. 0 grau de afinidade é uma medida da diferença entre a hierarquia dos val ores de dois indivíduos.

Assim, se os indivíduos tiverem uma hierarquia de valores semel hante, há uma maior probabilidade de se aproximarem geograficamente. 0 grau de afinidade representa aprimoramento relevante em relação ao model o de Zinchenko et al . (2003), uma vez que a afinidade de valores é importante para uma maior ou menor aproximação entre os indivíduos.

A despeito de a opinião sobre um indivíduo ser relevante para o interesse de um indivíduo se conectar a outro, a existência de afinidade de valores pessoais pode conduzir a uma tentativa de conexão que, no modelo, pode ser denotada pela aproximação geográfica. A diminuição das distâncias entre indivíduos conectados fortalece o vínculo das conexões, pois torna menos provável que a ligação se desfaça. De modo contrário, indivíduos não conectados podem se distanciar com uma probabilidade arbitrariamente estabelecida no mecanismo de simulação por Prob Afast' $_{\text {' }}$ que é corrigida em função do grau de afinidade.

\section{Processo iterativo de simulação}

Define-se um número $L$ de iterações do modelo. As iterações do algoritmo são análogas, dentro de certos limites, ao fator tempo. A cada iteração do processo de simulação, opiniões de i sobre j são ajustadas em função das conexões estabelecidas, das opiniões próprias e das opiniões de terceiros. N ovas conexões são realizadas e antigas conexões são desfeitas em função dos escores ajustados e do novo posicionamento dos indivíduos no espaço de duas dimensões.

Vale destacar que o processo iterativo é importante para que as opiniões sejam propagadas dentro da rede de relacionamento individual até que se estabeleçam as conexões na sociedade como um todo. Dependendo dos parâmetros do modelo de simulação, espera-se obter diferentes configurações de rede. A Figura 1 mostra o fluxograma simplificado do al goritmo de simulação implementado.

Deve-se destacar, portanto, que as opiniões latentes ou iniciais sofrem al terações em função da interação de um indivíduo com outros agentes de sua rede de relacionamento. 0 processo iterativo permite que vários indivíduos propaguem opiniões mesmo que não haja inicial mente uma conexão direta. Dependendo dos parâmetros da simulação, refletindo diferentes tipos de empresas, ou diferentes características das pessoas, os escores ou as opiniões podem privilegiar as competências ou os valores dos indivíduos. A seguir são discutidos os resultados da simulação.

\section{SIMULAÇÃO E RESULTADOS DO MODELO}

Pela equação referente à opinião latente, pode-se identificar que o parâmetro de consenso representa um peso correspondente à competência. No processo de definição da opinião latente, quanto maior o consenso, maior o peso que cada indivíduo atribui à variável referente às competências e menor o peso atribuído à variável referente às diferenças de valores entre os indivíduos. A seguir, estuda-se a influência do consenso na formação de redes sociais.

\section{Empresas ou atividades em que 0 consenso sobre as competências é nulo}

$N$ as empresas em que a competência seja difícil de identificar ou mensurar, as opiniões podem se formar principalmente em função dos aspectos ligados aos valores. Em um caso extremo, quando não há um consenso referente às competências, então 0 parâmetro 
consenso é igual a zero. N essa situação, o escore atribuído a um indivíduo decorre principalmente das diferenças de valores pessoais e do parâmetro de ruído de percepção de valores Erro $_{\mathrm{v}}$. 0 parâmetro de tolerância surge como importante mecanismo para a definição de opiniões. Quanto maior a tolerância, menor o impacto negativo na opinião de uma diferença de valores el evada entre os indivíduos.

Rodando 0 al goritmo descrito resumidamente no fluxograma da Figura 1, obtém-se, após as iterações, a seguinte configuração de rede, apresentada na Figura 2.

As estruturas $A, B$ e $C$, descritas na Figura 2, sim- plesmente representam leiautes diferentes da topologia de uma mesma rede. Quando uma linha sai de um indivíduo i e chega a outro j, existe uma conexão de i para j, quando a seta aponta para j. Assim, a seta denota o sentido da conexão, sendo necessária na apresentação da rede, uma vez que o relacionamento não é recíproco, ou seja, a matriz de relacionamentos não é simétrica. 0 gráfico $D$ da Figura 2 mostra simplesmente a dispersão geográfica dos indivíduos após as iterações simuladas. Quanto maior a dispersão, menor o nível de conexão, ou maior a distância entre os agentes.

Os resultados da simulação mostram que, quando 0

Figura 1 - Fluxograma sintético do algoritmo de simulação da dinâmica de formação de redes

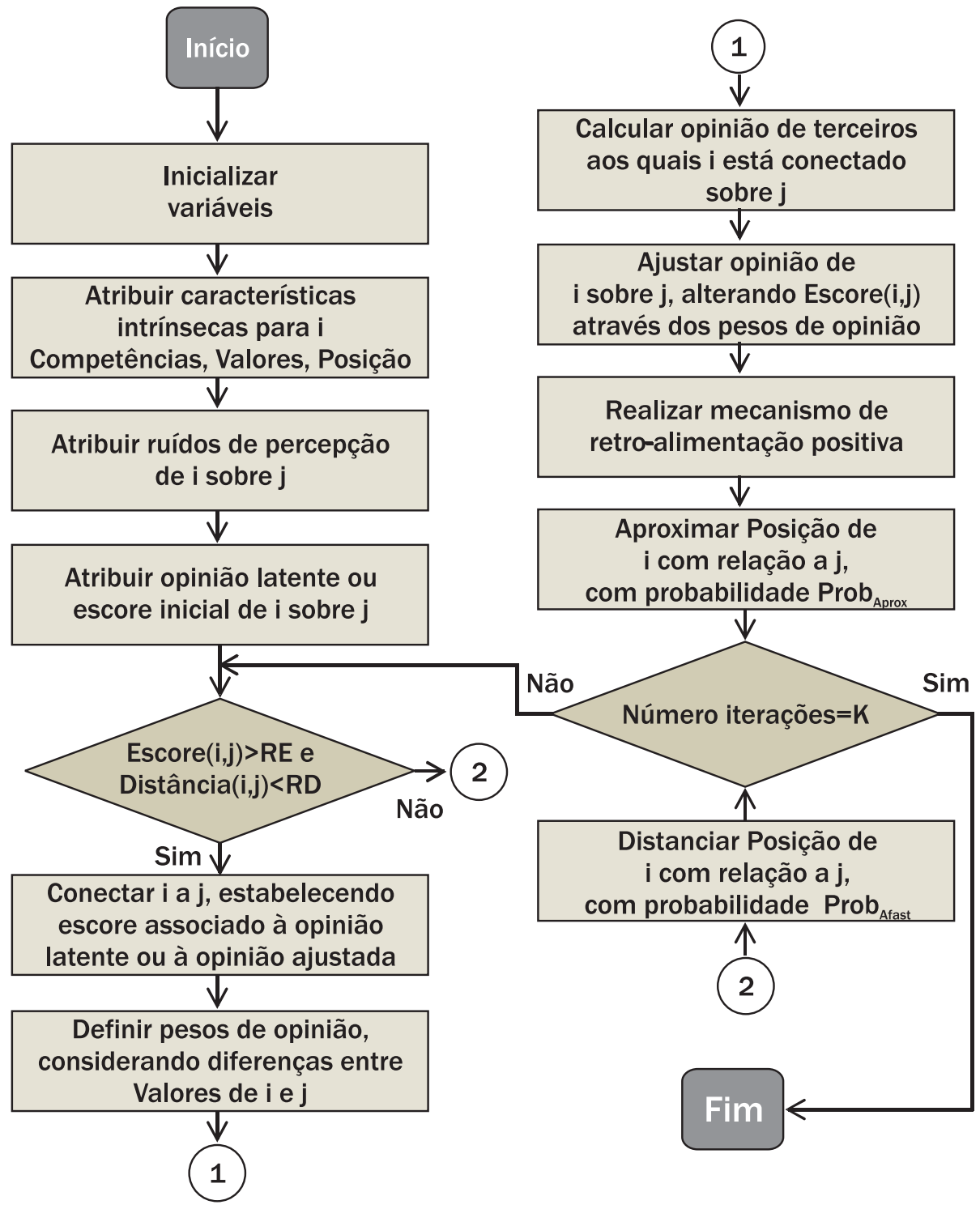


consenso sobre as competências dos indivíduos é nulo, poucas conexões são formadas e a rede é pouco densa. As conexões são, portanto, decorrentes principalmente das percepções sobre a diferença de valores pessoais. A estrutura denotada por A está associada a uma configuração circular, na qual simplesmente os indivíduos são colocados em uma roda e as conexões, estabelecidas como cordas de uma circunferência. Portanto, as conexões sempre estarão contidas na circunferência. Essa configuração permite uma visualização mais fácil do número de conexões, e eventualmente da centralização ou concentração das conexões.

É importante observar que, quando o consenso sobre as competências é baixo, diversos indivíduos não se conectam nem estão conectados. Q uando se privilegiam opiniões baseadas simplesmente em valores, a integração no grupo é baixa. Vários indivíduos ficam isolados nessa simulação.

A estrutura B, de spring embedding, permite identificar alguns núcleos para os quais diversas setas são direcionadas. 0 mecanismo de spring embedding é uma maneira de ordenamento de dados, não linear, para representar esteticamente os nós de uma rede baseandose em conceitos físicos. Supondo que as ligações entre os nós representem molas, a idéia básica do mecanismo é estabelecer uma configuração visual que implique uma menor energia para manter os nós interligados (Battista et al., 1994). Destaca-se que, em certa extensão, esse mecanismo é semel hante à técnica de splines cúbicos utilizada para a interpolação de dados.

Nessa simulação parecem existir alguns poucos indivíduos que têm uma maior rel evância no grupo. Apesar de terem um maior número de indivíduos conectados a eles, a sua influência é limitada, pois o número de conexões é baixo. A estrutura $C$ visa evidenciar os principais agentes da rede, apresentando uma maior aglomeração de indivíduos que possuem uma maior rede de relacionamentos. 0 gráfico $D$ mostra a distribuição no espaço bidimensional das posições dos indivíduos após as iterações. 0 posicionamento geográfico dá pistas de que não há uma melhoria na aproximação dos indivíduos, uma vez que a distribuição ainda é dispersa. No caso em que o consenso é nulo, portanto, pequenos grupos aglutinam-se em torno de poucos indivíduos, denotando a existência de núcleos com pouca influência, que não interagem fortemente.

\section{Empresas ou atividades em que o consenso sobre as competências é total}

Contrapondo-se à situação anterior, em organizações em

Figura 2 - Resultados da topologia e da dispersão da rede para o caso de consenso igual a 0,0

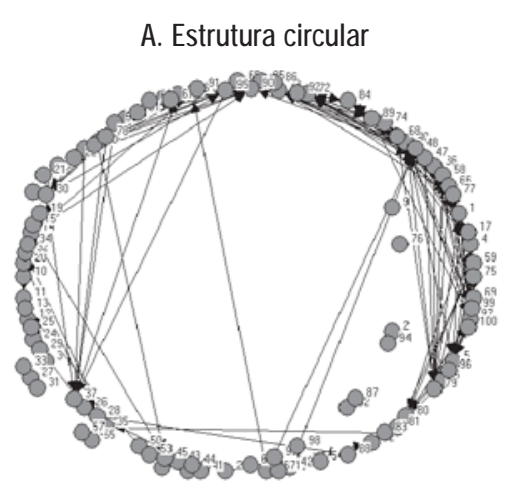

C. Componentes principais

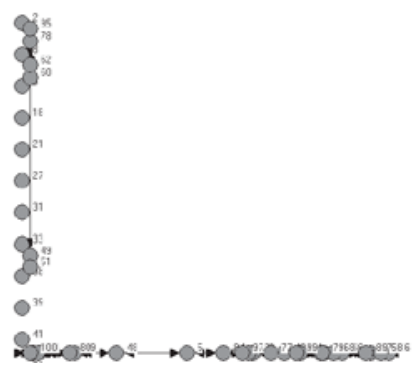

B. Spring embedding

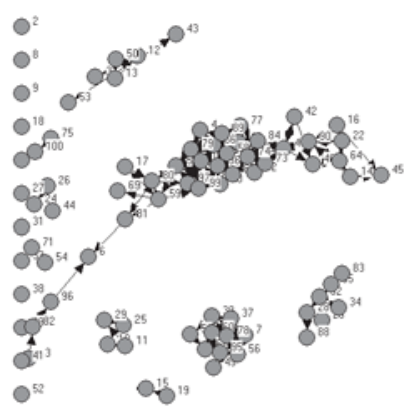

D. Mapa de posicionamento

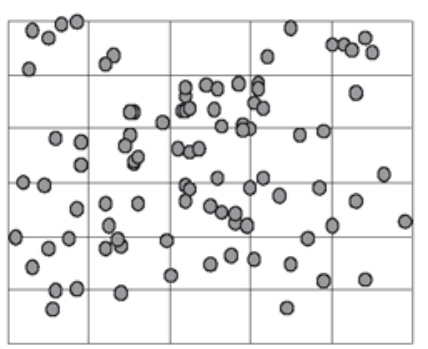


que as competências são fáceis de identificar, ou de mensurar, o parâmetro consenso é elevado. Considerando 0 caso extremo em que o consenso é total e igual a 1, os indivíduos atribuem opiniões baseando-se unicamente na percepção sobre as competências dos outros. Essa percepção está sujeita a um ruído denotado pelo parâmetro de Erro $_{H}$. A opinião que os agentes fazem sobre os outros não leva em consideração os valores dos individuos. Por exemplo, estudos empíricos podem envolver respostas a questionários que, eventual mente, podem não representar adequadamente os constructos de interesse. Mais ainda, as condições de aplicação do questionário podem ser pouco controladas, implicando 0 aumento da variabilidade da variável observada e prejudicando a significância dos resultados. Adicional mente, observações empíricas podem ser realizadas em escala inadequada para a utilização de várias técnicas estatísticas. A Figura 3 resume os resultados da simulação.

Destaca-se, no caso de consenso total, que a rede se torna muito mais interligada e densa. Pela anál ise da estrutura circular A, pode-se identificar que todos os indivíduos conectam-se a algum outro. Mesmo aqueles que não têm nenhuma seta apontando para eles, possuem setas saindo deles, denotando seu interesse em estar li- gados a alguns indivíduos. A concentração de setas implica uma região em que a estrutura é mais central izada, denotando a existência de agentes aos quais os indivíduos se conectam com mais freqüência e que podem ser considerados agentes de grande influência no grupo.

A estrutura $C$ de componentes principais permite evidenciar uma aglomeração de agentes que têm mais conexões. A estrutura B mostra também a existência de concentração das conexões em alguns indivíduos, bem como a conexão de todos os indivíduos à rede. Mesmo os indivíduos aos quais outros agentes atribuem escores baixos buscam se conectar. Os indivíduos aos quais são atribuídos menores escores estão evidenciados na parte mais externa da estrutura $B$.

Na configuração da simulação, a dispersão geográfica dos indivíduos também é menor, em comparação com a dispersão dos indivíduos no caso em que o consenso é nulo. Observa-se não somente uma concentração em função dos escores, como também uma concentração geográfica no espaço bidimensional, podendo refletir uma maior aproximação entre os indivíduos, pois nenhum agente está isolado. A competência se torna importante fator de aproximação dos indivíduos. Assim, quando a opinião é formada por aspectos obje-

Figura 3 - Resultados da topologia e da dispersão da rede para 0 caso de consenso igual a 1,0

\section{A. Estrutura circular}

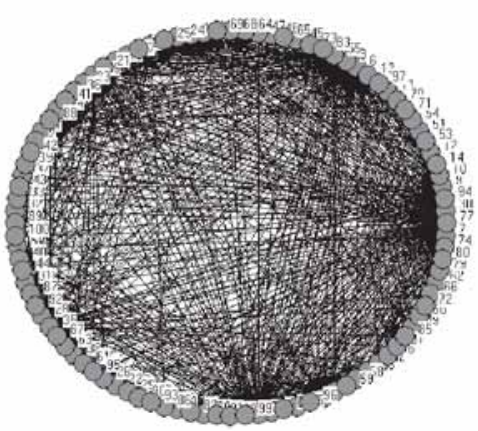

C. Componentes principais

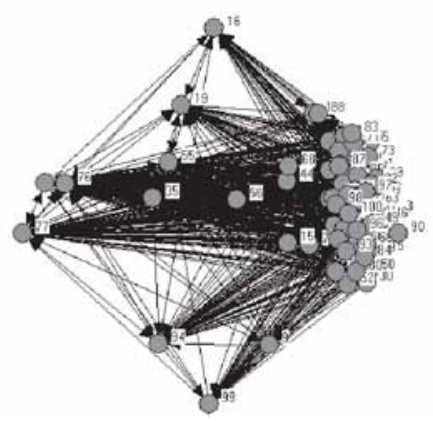

\section{B. Spring embedding}

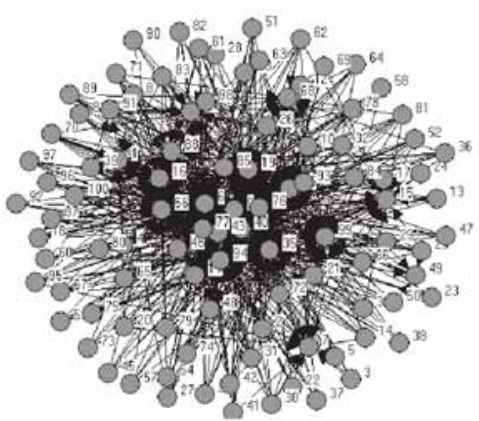

D. Mapa de posicionamento

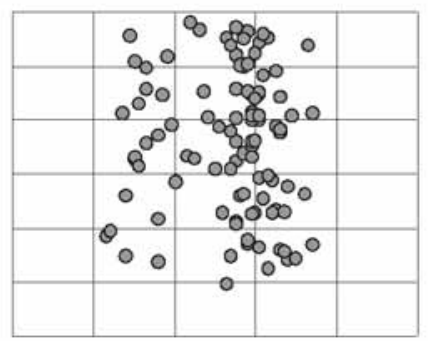


tivos ligados unicamente às competências dos indivíduos, a rede formada parece ser mais densa.

\section{Outros resultados do modelo}

Como é de se esperar, o caso mais comum, em que o consenso está entre 0 e 1 , implica uma situação intermediária à discutida nos itens anteriores. Assim, os indivíduos, ao formularem suas opiniões latentes ou escores iniciais, ponderam com os aspectos objetivos e subjetivos associados. A Figura 4 apresenta os resultados em que o consenso fica em um patamar de 0,5.

Assim, a opinião possui um componente de percepção de competências e de valores. Pode-se observar um maior número de conexões entre os indivíduos, em comparação ao caso em que o consenso é nulo. Há a formação de aglomeração de alguns el ementos que não se conectem à rede. Tendo em vista que, nessa situação, não somente as competências como também os valores têm relevância no estabelecimento dos escores, então os grupos podem se diferenciar em função dos valores e, dessa forma, criar núcleos cuja coesão pode ser menor.

Comparativamente ao caso em que o consenso é nulo, as distâncias diminuem, porém ainda não há uma aproximação tão grande quando se compara com 0 nível de agregação no espaço do caso de consenso total. Assim, os indivíduos buscam naturalmente uma maior aproximação, porém questões subjetivas ligadas às diferenças entre os valores ainda representam barreiras para um maior contato.

Pode-se também investigar o impacto dos ruídos de percepção na estrutura das redes formadas. Considerando o caso em que a opinião depende em proporções iguais dos aspectos de competências e de valores, obtêm-se as configurações de rede dadas na Figura 5, alterando-se o nível da variância dos erros nas simulações.

Os resultados mostram que a rede é mais densa quando os erros na percepção das competências são mais baixos, e quando os erros na percepção dos valores individuais são altos. Finalmente, é interessante observar também o comportamento do modelo em grupos em que o número de indivíduos é baixo. Considerando, por exemplo, $N=10$, o consenso pode ser fator determinante da estruturação, ou não, de conexões. A Figura 6 mostra os resultados de simulações para consenso baixo e para alto, evidenciando que, quando as competências são mais enfatizadas no processo de formação de opinião, é mais provável a exis-

Figura 4 - Resultados da topologia e da dispersão da rede para o caso de consenso igual a 0,5

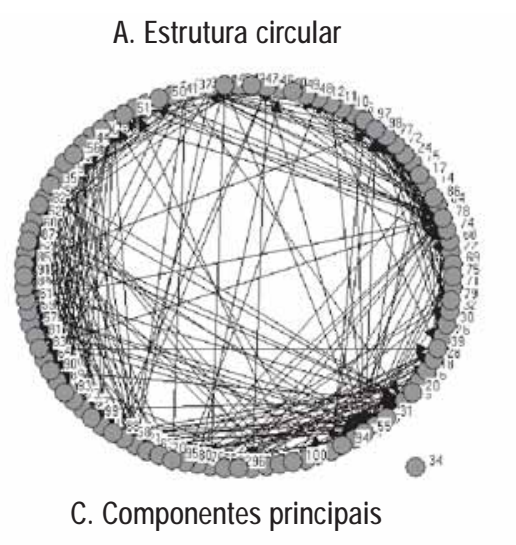

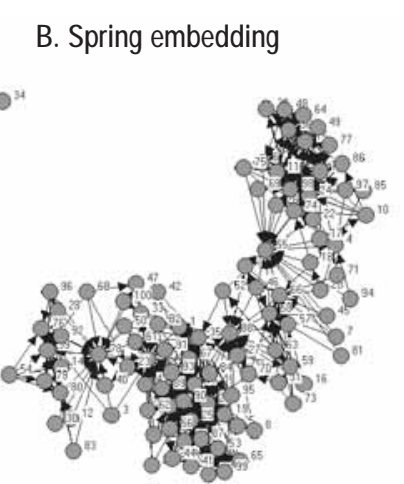

D. Mapa de posicionamento

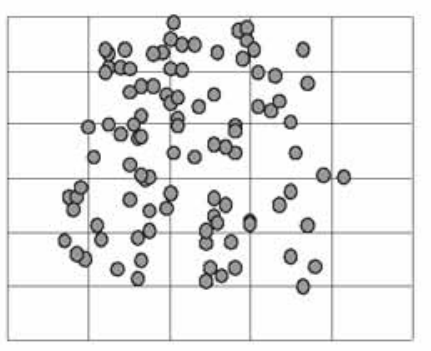


tência de indivíduos com capacidade de influenciar o grupo. Em geral, quando o consenso é alto, dado o menor número de indivíduos, há uma mai or centralização da rede. Grupos com um número pequeno de indivíduos são de fato mais simples de gerenciar.

Quando o consenso é baixo, observa-se uma grande probabilidade de não se formarem conexões, e a empresa não se beneficiar de sinergias entre os indivíduos. Quando os valores individuais têm um peso grande na definição de opinião, em um grupo com poucos agentes, há uma tendência de os indivíduos se isolarem. Nesse contexto, os gestores podem, de certa maneira, fomentar conexões estabelecendo uma estrutura organizacional que privilegie as competências.

Figura 5 - Densidade da rede em função da variância do ruído das competências e dos valores

Baixo ruído na percepção de Competências

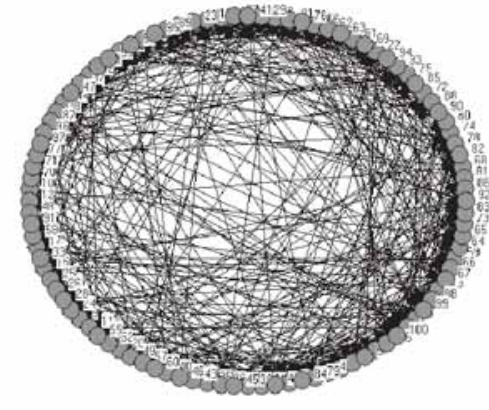

Baixo ruído na percepção de Valores

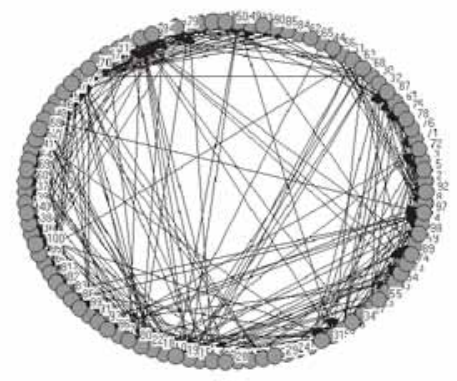

Alto ruído na percepção de Competências

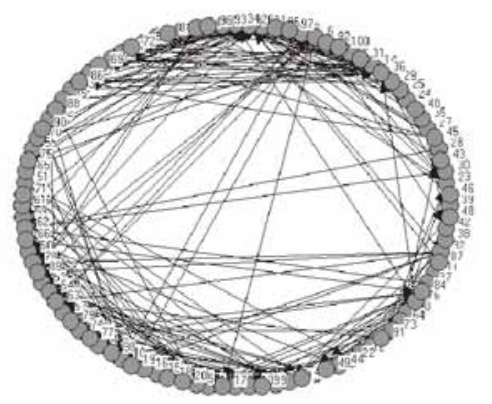

Alto ruído na percepção de Valores

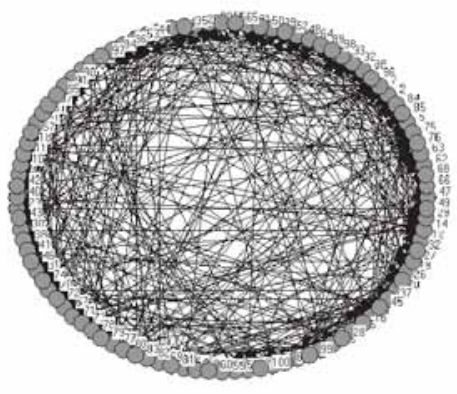

Figura 6 - Impacto do consenso em sociedades com poucos indivíduos

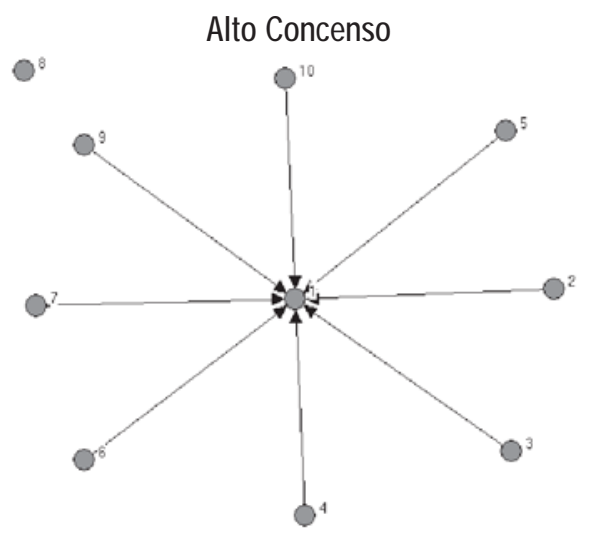

$54 \cdot$ - RAE - VOL. $46 \cdot N 03$ 


\section{COMENTÁRIOS FINAIS}

0 processo de simulação permite que se crie um ambiente artificial no qual se podem avaliar influências específicas de parâmetros do modelo. Apesar de a modelagem apresentar uma parametrização consistente com vários aspectos relativos a competências e valores na formação de conexões em redes sociais, não leva em consi deração a sutileza e não esgota a compl exidade da formação de conexões nas interações humanas.

Por exemplo, o modelo define que as conexões estão associadas à opinião positiva que se tem de outro indivíduo. Dessa forma, há sempre um viés no estabelecimento das conexões, pois não se prevêem possíveis conexões negativas. Além disso, a formulação do escore, ou da opinião latente, envolve uma combinação linear de competências e val ores que eventual mente pode não ser adequada. As distribuições de probabilidade das variáveis aleatórias associadas às competências, aos valores, aos ruídos de percepção e ao mecanismo de real imentação podem não representar adequadamente as características dos grupos. Porém, a despeito das limitações de um modelo simplificado, consegue-se filtrar as várias influências e identificar diversos pontos importantes que podem ser utilizados para refletir sobre o papel das competências e valores na formação de redes sociais e suas prováveis implicações para a gestão das organizações.

Os resultados encontrados evidenciam, no modelo simulado, que as competências consistem num fator de aproximação e de facilitação de conexões nas redes sociais, já que quando o consenso de competências é nulo as conexões são mais difíceis de se formar. Em contrapartida, quando o consenso é total, a rede é interligada e densa, apresentando um maior número de conexões, destacando-se indivíduos que aglutinam os demais.

Esses resultados remetem ao conceito de Zarifian (2001), para quem as competências são desenvolvidas e exercidas em situações de interação social. Pode-se vislumbrar que um consenso alto em torno das competências de um indivíduo, que acaba por se manifestar no número de conexões com el e estabelecidas, pode atuar como el emento reforçador dessas mesmas competências. Quanto mais os indivíduos se percebem competentes, tendo como parâmetro as conexões com ele estabelecidas, mais tenderão a manifestar as suas competências, que, por sua vez, podem estimular 0 consenso e a densidade das redes.

Esses resultados sugerem que, quando se pretende estimular a formação de redes sociais nas organiza- ções, em situações em que os indivíduos não se conhecem, as competências devem ser privilegiadas com critério, caso se deseje que as redes se tornem densas e com grande número de conexões.

Quando a opinião que os indivíduos formam é baseada principalmente nos valores pessoais, surgem indivíduos isolados, podendo haver uma tendência de formação de grupos antagônicos. Isso pode ocorrer devido ao fato de as dimensões de valores possuírem pólos opostos, priorizados de forma diferente pel os indivíduos de um mesmo grupo, conforme indica a teoria de valores de Schwartz (1992). Entretanto, os resultados encontrados podem ser decorrentes do fato de o modeIo não ter contemplado adequadamente um parâmetro de tempo no processo de formação das conexões, levando a perceber os valores como exercendo uma influência menos aglutinadora, já que percebê-los exige tempo de convivência e a oportunidade de observar processos de tomada de decisão entre alternativas conflitantes, conforme sugere Schwartz (1992). A pesar de as iterações do algoritmo representarem unidades de tempo discretas na simulação, podem não refletir a sutileza das interações entre os indivíduos, uma vez quea opinião pode ser continuamente reformulada.

Um dos resultados revelados pela simulação do modelo se refere ao ruído na percepção de competências e valores. Quando existe um baixo ruído na percepção das competências, as redes mostram-se densas, ao contrário de quando o ruído é el evado. Porém, o mesmo não ocorre quanto à influência do erro na percepção de valores; neste caso, os resultados revelaram que, sendo o ruído baixo, a rede revela-se menos densa.

Esse resultado com relação aos valores não encontra apoio na literatura pertinente, seja ela relativa à teoria de valores ou à congruência entre os val ores dos indivíduos, onde esta é entendida como fator de estímulo à compatibilidade entre os indivíduos e seus grupos de trabal ho (Kristof-Brown, Zimmerman e Johnson 2005), assim como contraria o senso comum. Tratase, portanto, de um resultado intrigante, que deverá ser mais bem examinado, antes mesmo de se recorrer a testes empíricos do modelo. Em contrapartida, é importante considerar que a complexidade de interações entre os indivíduos pode gerar resultados pouco intuitivos, e, neste contexto, a modelagem pode ser útil por possibilitar a formulação de hipóteses de relacionamentos não lineares que dificilmente poderiam ser imaginadas pelo senso comum.

Uma agenda para futuras pesquisas deve incluir teste empírico do modelo em ambientes organizacionais 
diversos, visando comparar o comportamento das variáveis estabelecidas no model o com o comportamento delas em situação real de funcionamento das redes sociais. Concordando com Harrison e Carroll (2002) e Macy e Willer (2002), a modelagem, simulação e observação empírica da realidade podem ser entendidas como um conjunto de possibilidades de entendimento do mundo real que se complementam. No entanto, os métodos computacionais fornecem, com seus modelos, al gumas conclusões, assim como orientações e sugestões para pesquisas empíricas.

Neste estudo, o model o desenvolvido e anal isado permitiu reflexões e conclusões importantes tanto para a geração de teoria quanto para a prática organizacional. Para que o modelo seja testado em uma realidade empírica, é necessária uma adequada operacionalização dos conceitos de valores e competências, e que o estudo seja orientado por teorias de valores e competências compatíveis entre si, assegurando a consistência teórica.

\section{REFERÊNCIAS BIBLIOGRÁFICAS}

ABRAHAMSON, E.; ROSENKOPF, L. Social network effects on the extent of innovation diffusion: a computer simulation. Organization Science, v. 8 , n. 3, p. 289-309, 1997.

ANCONA, D. G.; CALDWELL, D. F. Demography and design: predictors of new product team productivity. Organizational Science, v. 3, n. 3, 321341, 1992.

ARMENGOL, A. C.; JACKSON, M. O. The effects of social networks on employment and inequality. The American Economic Review, v. 94, n. 3, p. 426-462, 2004.

BATTISTA, G.; EADES, P.; TAMASSIA, R.; TOLLIS, I. G. Algorithms for drawing graphs: an annotated bibliography. Computational Geometry: Theory and Applications, v. 4, n. 5, p. 235-282, 1994.

BERMAN, E. M.; WERTHER JR., W. Broad-based consensus building. International Journal of Public Sector M anagement, v. 9, n. 3, p. 61-72, 1996.

BORGATTI, S. The state of organizational social network research today. Department of Organization Studies: Boston University, 2003. mimeo.

BORGATTI, S.; FOSTER, P. The network paradigm in organizational research: a review and typology. Journal of M anagement, v. 29, n. 6, p. 9911013, 2003.

BORGATTI, S.; MOLINA, J. Ethical and strategic issues in organizational network analysis. Journal of Applied Behavioral Science, v. 39, n. 3, p. 337 350, 2003.

BRASS, D. J. Being in the right place: a structural analysis of individual influence in an organization. Administrative Science Quarter, v. 29, p. 518529, 1984.
CARIOLA, H. M. L.; QUIROZ, M. A. M. Competencias generales, competencias laborales y currículum. In: NOVICK, M.; GALLART, M. A. Competitividad, redes productivas y competencias laborales. Montevideo: Cinterfor, 1997.

COLEMAN, J. S. Social capital in the creation of human capital. American Journal of Sociology, v. 94, n. 5, p. 95-120, 1988.

COLLIER, P. Social Capital and Poverty. Washington, DC: The World Bank, 1998.

CUMMINGS, J. N. Work groups, structural diversity, and knowledge sharing in a global organization. Management Science, v. 50, n. 3, p. 352383, 2004.

DUTRA, J. S. Competências: conceitos e instrumentos para a gestão de pessoas na empresa moderna. São Paulo: Atlas, 2004.

FLEURY, A.; FLEURY, M. T. Estratégias empresariais e formação de competências. São Paulo: Atlas, 2001.

FOMBRUN, C. J. Strategies for network research in organizations. Academic of Management Review, v. 7, n. 2, p. 280-291, 1982.

GRAN OVETTER, M. Economic action and social structure: the problem of embeddedness. American Journal of Sociology, v. 91, n. 3, p. 481-510, 1985.

HARRISON, J. R.; CARROLL, G. R. The dynamics of cultural influence networks. Computational and Mathematical Organization Theory, v. 8, n. 1, p. 5-30, 2002.

HERBSLEB, J. D.; MOCKUS, A. An empirical study of speed and communication in globally distributed software development. IEEE Transactions on Software Engineering, v. 29, n. 6, p. 481-494, 2003.

JONES, C.; HESTERLY, W.; BORGATTI, S. A general theory of network governance: exchange conditions and social mechanisms. Academy of M anagement Journal, v. 22, n. 4, p. 911-945, 1997.

KRISTOF-BROWN, A.; ZIMMERMAN, R.; JOHNSON, E. Consequences of individual's fit at work: a meta-analysis of person-job, personorganization, person-group, and person-supervisor fit. Personnel Psychology, v. 58, n. 2, p. 281-342, 2005.

LE BOTERF, R. Desenvolvendo a competência dos profissionais. Porto Alegre: Artmed, 2003.

LEANA, C.; BUREN III, H. Organizational social capital and employment practices. The Academy of Management Review, v. 24, n. 3, p. 538-555, 1999.

LEENDERS, R. ENGELEN, J., KRATZER, J. Virtuality, communication, and new product team creativity: a social network perspective. Journal of Engineering and Technology M anagement, v. 20, p. 69-92, 2003.

MACY, M. W.; WILLER, R. From factors to actors: computational sociology and agent-based modeling. Annual Review of Sociology, v. 28, p. 143-166, 2002.

MCLAGAN, P. A. Competencies: the next generation. Training \& Development, v. 51, n. 5, p. 40-47, 1997.

MCLEOD, J. M.; CHAFFEE, S. H. Interpersonal Approaches to Communication Research. The American, v. 16, n. 4, p. 469-499, 1973. 
MILGRAM, S. The small world problem. Psychology Today, v. 22, p. 61-67, 1967.

MURPHY, K. M.; SHLEIFER, A. Persuasion in politics. The American Economic Review, v. 94, n. 2, p. 435-446, 2004.

PODOLNY, J. M.; BARON, J. N. Resources and relationships: social networks and mobility in the workplace. American Sociological Review, $v$. 62, n. 5, p. 673-693, 1997.

PORTES, A.; SEN SEN BREN NER, J. Embeddedness and immigration: notes on the social determinants of economic action. American Journal of Sociology, v. 98, n. 6, p. 1320-1350, 1993.

PUTNAM, R. The prosperous community-social capital and public life. American Prospect, v. 4, n. 13, p. 35-42, mar. 1993.

RAUCH, J. E. Business and social networks in international trade. Journal of Economic Literature, v. 39, n. 4, p.1177-1203, 2001.

REAGANS, R.; ZUCKERMAN, E. W. N etworks, diversity, and productivity: the social capital of corporate $R \& D$ teams. Organization Science, v. $12, n$. 4, p. 502-517, 2001.

RUAS, R. L. Gestão por competências: uma contribuição à estratégia das organizações. In: RUAS, R. L.; ANTONELLO, C. S.; BOFF, L. H. Os novos horizontes da gestão: aprendizagem organizacional e competências. Porto Alegre: Bookman, 2005.

SCHWARTZ, S. H. Universals in the content and structure of values: Theoretical advances and empirical tests in 20 countries. In: ZAN NA, M. (Ed.). Advances in Experimental Social Psychology, v. 25, p. 1-65, New York: Academic Press, 1992.
SORENSON, O. Social networks and industrial geography. Journal of Evolutionary Economics, v. 13, n. 5, p. 513, 2003.

SPARROWE, R. T.; LIDEN, R. C.; KRAIMER, M. L. Social networks and the performance of individuals and groups. Academy of M anagement Journal, v. 44, n. 2, p. 316-325, 2001.

SUSSKIND, L. E. An alternative to Robert's rules of order for groups, organizations and ad hoc assemblies that want to operate by consensus. In: The Consensus Building Handbook: a comprehensive guide to reaching agreement. Sage Publications, 1999.

WEJNERT, B. Integrating models of diffusion of innovations: a conceptual framework. Annual Review of Sociology, v. 28, n. 1, p. 297-326, 2002.

WIRTH, L. Consensus and mass communication. America Sociology Review, v. 13, p. 1-15, 1948.

WOOLDRIGE, B.; FLOYD, S. W. Strategic process effects on consensus. Strategic Management Journal, v. 10, n. 3, p. 295-302, 1989.

ZARIFIAN, P. 0 modelo da competência: trajetória histórica, desafios atuais e propostas. São Paulo: Editora Senac São Paulo, 2003.

ZARIFIAN, P. O bjetivo competência: por uma nova lógica. São Paulo: Atlas, 2001.

ZINCHENKO, E; STEINBOCK, D. J.; MILLER, J.; THOMAS, R, J. When dissent is good: the interplay between reputation and social networks in an artificial society. Santa Fe Institute Letters on Complex Systems, 2003. Disponível em <http://www.sonic.net/\%7Edaniel/pubs/steinbockdissent.pdf>. Acessado em 15 dez. 2004.

\section{Artigo recebido em 20.12.2004. Aprovado em 27.04.2006.}

\section{Herbert Kimura}

Professor da Universidade Presbiteriana M ackenzie. Doutor em Administração pela USP.

Interesses de pesquisa nas áreas de Derivativos, Criação de Valor, Finanças Comportamentais

e Finanças Corporativas.

E-mail: hkimura@mackenzie.com.br

Endereço: Rua da Consolação, 896, 70 andar, sala 76, Consolação, São Paulo - SP, 01302-907.

\section{Maria Luisa Mendes Teixeira}

Professora da Universidade Presbiteriana Mackenzie. Doutora em Administração pela USP. Interesses de pesquisa nas áreas de Gestão Baseada em Valores, Valores e Mudança Organizacional, Axiomas Sociais e Organizações.

E-mail: mallu@mackenzie.com.br

Endereço: Rua da Consolação, 896, 70 andar, sala 76, Consolação, São Paulo - SP, 01302-907.

\section{Arilda Schmidt Godoy}

Professora da Universidade Presbiteriana Mackenzie. Doutora em Educação pela USP. Interesses de pesquisa nas áreas de Aprendizagem Individual, Aprendizagem nas Organizações, Desenvolvimento de Competências.

E-mail: arilda@mackenzie.com.br

Endereço: Rua da Consolação, 896, 70 andar, sala 76, Consolação, São Paulo - SP, 01302-907. 